\title{
Selective bronchial intubation for pulmonary emphysema
}

\author{
A N CAMPBELL, Y ZARFIN, AND M PERLMAN \\ Division of Perinatology, Hospital for Sick Children, Toronto, Canada
}

SUMmaRY Two neonates with respiratory distress syndrome developed unilateral pulmonary interstitial emphysema causing mediastinal shift and compressive atelectasis. Treatment with contralateral bronchial intubation for five days proved to be life saving.

Pulmonary interstitial emphysema in neonates may occur spontaneously or may be seen as a complication of hyaline membrane disease. The interstitial air may dissect along the peribronchial and perivascular spaces causing a pneumomediastinum or pneumothorax. Continuing accumulation of interstitial air in a lung may lead to the formation of bullae causing mediastinal shift and compression of the contralateral lung. This may cause further respiratory embarrassment to a neonate already in critical respiratory failure. Several successful forms of treatment including lobectomy ${ }^{1}$ and selective bronchial intubation have been reported. ${ }^{23}$

We report two infants with severe unilateral pulmonary interstitial emphysema whose symptoms and radiographic appearances resolved dramatically after selective bronchial intubation.

\section{Case reports}

Case 1. A girl weighing $1400 \mathrm{~g}$ was born at 32 weeks' gestation by caesarean section. Her Apgar scores were 2 at one minute and 4 at five minutes. She rapidly developed respiratory distress requiring tracheal intubation and mechanical ventilation and was transferred to our neonatal intensive care unit at age 4 hours. On day 2 , she developed bilateral pneumothoraces, although maximal peak inspiratory pressure had not exceeded $25 \mathrm{~cm} \mathrm{H}_{2} \mathrm{O}$. These were drained by chest tubes with underwater seals. With subsequent improvement her need for oxygen treatment was reduced $\left(\mathrm{F}_{\mathrm{I}} \mathrm{O}_{2} \quad 0 \cdot 3\right.$ by day 7$)$. Pulmonary interstitial emphysema of the left lung was noted at this time on a radiograph but in view of her good clinical status she was extubated next day and maintained on nasopharyngeal continuous positive airways pressure until day 11 . Over the succeeding days the left pulmonary interstitial emphysema worsened and by day 18 she required an $\mathrm{F}_{1} \mathrm{O}_{2}$ of $0 \cdot 8$ in a hood. A further left pneumothorax was drained $\stackrel{\mathbb{\Omega}}{\Omega}$ with little benefit and she was ventilated again. Onळ day 21 radiography showed massive left sided $\vec{\circ}$ pulmonary interstitial emphysema with herniation of the left lung into the right hemithorax with $\vec{\rho}$ mediastinal shift (Fig. 1). The $\mathrm{F}_{\mathrm{I}} \mathrm{O}_{2}$ was 0.9 and $\mathrm{PaCO}_{2}$ was $68 \mathrm{~mm} \mathrm{Hg}$. Vigorous chest physiotherapy? every hour for eight hours had no beneficial effect. of On day 22 her right main bronchus was selectively 6 intubated and within four hours a noticeable reduc-o tion in hyperinflation of the left lung was seen on: radiographs and the $\mathrm{F}_{1} \mathrm{O}_{2}$ had fallen to 0.45 (Fig. 2). 오 Twelve hours later her oxygen requirements again increased, despite radiological findings of relatively $\mathbb{\Phi}$ normal inflation of the left lung and re-expansion of the right lung. The endotracheal tube was with- $\frac{\mathbb{D}}{3}$ drawn above the carina with transient improvemento in oxygen requirement. Twelve hours later, a repeat ${ }_{-}^{-}$ chest radiograph showed the return of massige $\overrightarrow{0}$ pulmonary interstitial emphysema of the left lueg? + with collapse of the right lung. This was aga associated with increasing oxygen requirements. Selective intubation of the right main bronchus was

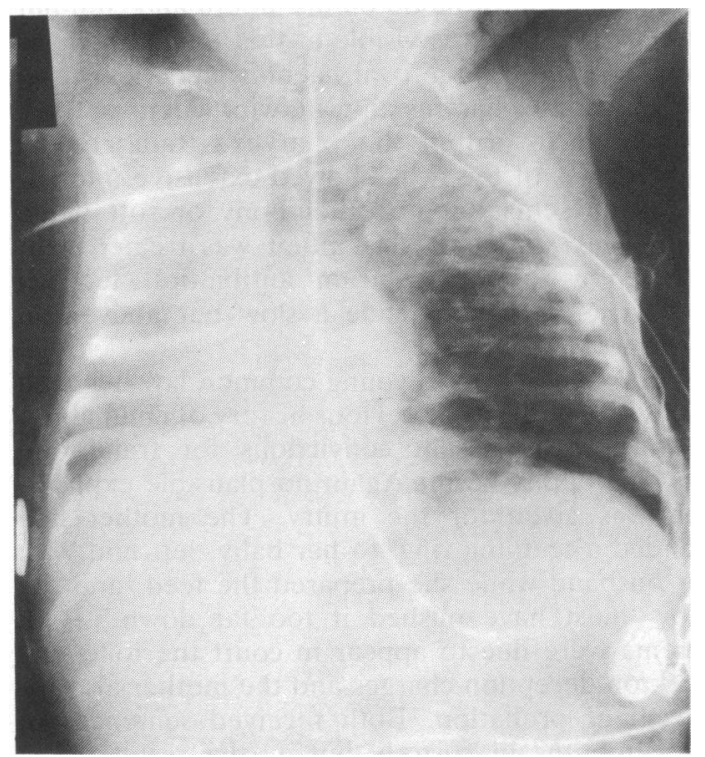

Fig. 1 Chest radiograph (case 1) on day 21 showing massive left pulmonary interstitial emphysema. 


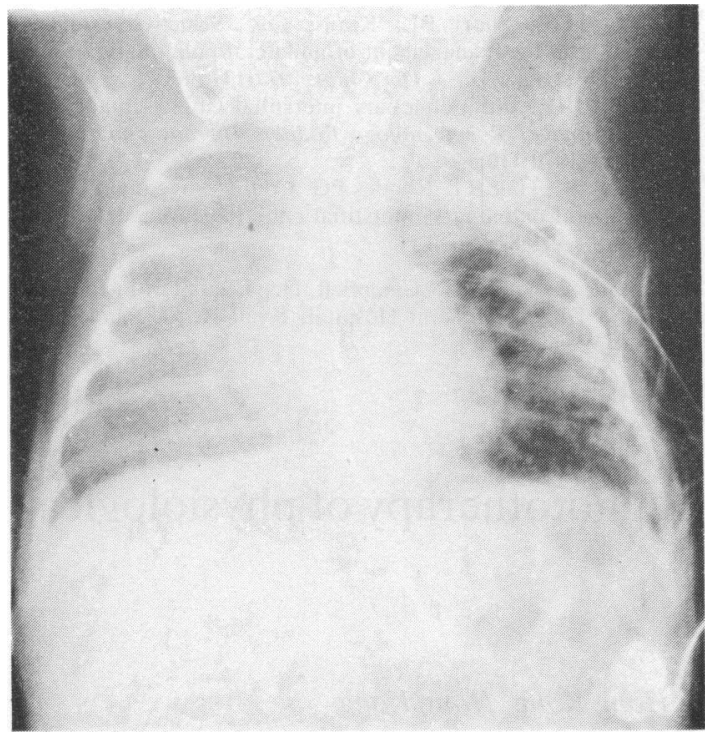

Fig. 2 Chest radiograph (case 1) on day 25 showing resorption of the pulmonary interstitial emphysema.

repeated, and again resulted in reduction of oxygen requirements. The tube was left in this position for an arbitrary period of five days with continued improvement. The endotracheal tube was then withdrawn to the trachea, and the infant was extubated three days later. She was weaned to room air on day 50 .

Case 2. A boy weighing $1460 \mathrm{~g}$ born by vaginal vertex delivery at 30 weeks' gestation was transferred to our unit at 2 hours of age because of respiratory distress. He rapidly required mechanical ventilation and a chest radiograph at 12 hours of age showed early signs of pulmonary interstitial emphysema. On day 2 a right tension pneumothorax was drained by a chest tube and underwater seal. His clinical status continued to deteriorate, however, and a chest radiograph showed worsening pulmonary interstitial emphysema. High frequency oscillation was attempted but failed after an initial clinical improvement. By day 5 chest radiographs showed massive left sided pulmonary interstitial emphysema with lung herniation causing acute respiratory embarrassment. The right main bronchus was selectively intubated and by 12 hours a good clinical and radiographic response was obtained-the endotracheal tube was then withdrawn above the carina. On day 8 , however, the left pulmonary interstitial emphysema recurred; selective intubation was repeated and the tube left in situ for five further days. Return of the tube to the trachea at this time caused no further setbacks and extubation was achieved after five days.

\section{Discussion}

Pulmonary interstitial emphysema may occur in up to $20 \%$ of infants with hyaline membrane disease and several methods of treatment have been described. ${ }^{4}$ Lobectomy may be hazardous in an infant already compromised by hyaline membrane disease and has the long term disadvantage of removing lung tissue which presumably has potential for recovery. Leonidas et $a l^{5}$ describe the use of chest percussion and vibration, postural drainage, tracheal suction, and assisted ventilation with $100 \%$ oxygen for five to 10 minutes every hour to enhance reabsorption of gas. This method involves a theoretical risk of retrolental fibroplasia and of pulmonary oxygen toxicity.

Dickman $^{2}$ and Brooks ${ }^{3}$ describe five neonates treated with selective intubation for pulmonary interstitial emphysema. The rationale for this form of treatment depends upon hyperexpansion of the intubated lung and resorption of air from the contralateral lung as a result of bronchial occlusion. The procedure of selective intubation of the right bronchus is relatively simple, while the left may be more difficult. The diameter of the tube used is the same as that used for tracheal intubation. The tube is simply inserted two to four cm further than usual with the bevel on the end of the tube positioned so that the long side is toward the main bronchus to be intubated. Turning the infant's head to the contralateral side may help in obtaining the correct position. Radiography must be performed to confirm the tube position. As the pulmonary interstitial emphysema improves, alteration in the peak pressures may be necessary to avoid hyperinflation and pneumothorax.

The duration of treatment is arbitrary. Initially in case 1 a dramatic improvement was followed by a deterioration and it may well be that obstruction of the right middle lobe bronchus as well as of the right upper bronchus occurred. We obtained a good result after five days in both of our patients while Brook's experience with four infants was from one and a half to five days. Complications that may occur are atelectasis of the right upper lobe, acute hypoventilation of the selectively intubated lung if the effective lung volume is too small, and the potential of bronchial injury with subsequent scarring and stenosis.

We would propose that the method of selective intubation is relatively straightforward to perform in a baby with pulmonary interstitial emphysema causing severe respiratory problems. The treatment may need to be continued for several days and the 
recurrence of pulmonary interstitial emphysema after the initial attempt should not preclude a second attempt.

\footnotetext{
References

1 Fletcher BD, Outerbridge EW, Youssef S, et al. Pulmonary interstitial emphysema in a newborn infant treated by lobectomy. Pediatrics 1974:54:808.

2 Brooks JG, Bustamante SA, Koops BL, et al. Selective bronchial intubation for the treatment of severe localised pulmonary interstitial emphysema in newborn infants. $J$ Pediatr 1977:91:648-52.
}

${ }^{3}$ Dickman GL, Short BL, Krauss DR. Selective bronchial intubation in the management of unilateral pulmonary interstitial emphysema. Am J Dis Child 1977;131:365.

4 Campbell RE. Intrapulmonary interstitial emphysema. American Journal of Roentgenology Radium Therapy and Nuclear Medicine 1970:110:449.

${ }^{5}$ Leonidas JC, Hall RT, Rhodes PG. Conservative management of unilateral pulmonary interstitial emphysema under tension. J Pediatr 1975;87:776-8.

Correspondence to Dr A N Campbell, Department of Paediatrics, Sunderland District General Hospital, Kyall Road, Sunderland, Tyne and Wear.

Received 3 April 1984

\title{
Serum bilirubin kinetics in intermittent phototherapy of physiological jaundice
}

\author{
S P LAU AND K P FUNG \\ Paediatric Department, Queen Mary Hospital, University of Hong Kong, Hong Kong
}

\begin{abstract}
SUMMARY Thirty four term babies with physiological jaundice were subjected to continuous phototherapy and to two regimens of intermittent phototherapy. The difference in serum bilirubin kinetics between the three groups of treated babies was insignificant; a schedule of one in four hours of irradiation achieved the same treatment effect as continuous phototherapy.
\end{abstract}

Over the past 20 years phototherapy has become the most common treatment for neonatal hyperbilirubinaemia. The question of subtle, long term side effects, however, has not been adequately answered and it is theoretically important, therefore, that infants should be subjected to the least amount of irradiation without sacrificing the overall efficiency of the treatment. Various schedules of intermittent phototherapy have been investigated. Because most earlier studies ${ }^{1-3}$ were concerned with the prophylaxis of hyperbilirubinaemia and treatment was started early without consideration of the individual variation of bilirubin kinetics, the results were not surprisingly controversial. Using a new mathematical approach to study the serum bilirubin kinetics in vivo this report compares the efficiency of three different regimens of phototherapy in jaundiced, term Chinese infants.

\section{Patients and methods}

Jaundiced term infants born in the Tsan Yuk
Maternity Hospital, Hong Kong with a birthweight of $2.5 \mathrm{~kg}$ or more were enrolled in the study. Infants with jaundice of known causes were excluded. When the serum bilirubin concentration reached 190 to $205 \mu \mathrm{mol} / \mathrm{l}(11.5$ to $12 \mathrm{mg} / \mathrm{dl})$ the babies were randomised into one of three groups receiving different regimens of phototherapy-group $A \equiv$ underwent continuous phototherapy; group B received four hours of phototherapy followed by four hours off, and group C underwent one hour of phototherapy and three hours off.

Phototherapy was administered by a bank of 8 fluorescent lamps (Duro-vita lite, $20 \mathrm{~W}$ ) in standard units. Irradiance was measured every morning by an IL 444 Radiometer (Spectrum 420-470 nanometer, International Light Inc, USA) at the centre of the mattress. A reading of $350 \mu \mathrm{W} / \mathrm{cm}^{2} \pm 5 \%$ was considered satisfactory and in case of deviation either the lamps were replaced or the distance between mattress and lamps was adjusted.

The total serum bilirubin concentration was measured 6 to 8 hourly by spectrophotometric technique (AO Bilirubinometer, American Optical Corporation). Phototherapy was stopped when the serum bilirubin concentration had fallen to 170 $\mu \mathrm{mol} / \mathrm{l}(10 \mathrm{mg} / \mathrm{dl})$. The change in the serum bilirubin concentration with time was represented by a bilirubin growth curve which was constructed by the least square method. ${ }^{4} \mathrm{~A}$ polynomial of third degree $\left(S B=a+b \times T+c \times T^{2}+d \times T^{3}\right.$ where $\mathrm{SB}=$ interpolated serum bilirubin concentration; $a, b, c, d=$ coefficients of the polynomial equation; and $\mathrm{T}=$ time) was found to be a satisfactory mathe- 